\title{
A Node Architecture for Disaster Relief Networking
}

\author{
Fokke Hoeksema, Marnix Heskamp, Roel Schiphorst, Kees Slump \\ University of Twente, \\ Department of Electrical Engineering, Mathematics and Computer Science (EEMCS) \\ Signals and Systems Group, \\ P.O. box 217 - 7500 AE Enschede - The Netherlands

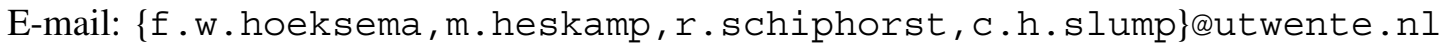

\begin{abstract}
In this paper we present a node architecture for a personal node in a cognitive ad-hoc disaster relief network. This architecture is motivated from the network system requirements, especially single-hop distance and jamming-resilience requirements.

It is shown that the power consumption of current-day Analog to Digital Converters (ADCs) limits the channel bandwidth that can be used, both for a baseband processing system and the scanning system. We therefore propose to use a form of Frequency Hopping Time Division Multiplexing (FH/TDD) system, similar to Bluetooth. However, we propose to use OFDM as modulation scheme instead of GFSK and show that the OFDM symbol length for the frequency range at hand $(400-1000 \mathrm{MHz})$ is consistent with a Bluetooth-alike FH/TDD scheme. We propose to modify the FH/TDD scheme to incorporate a radio-spectrum measurement phase.

Based on these measurements carriers in the OFDM-based scheme are selected in such a fashion that legacy users are avoided. In case of too much legacy-user spectral usage hopping patterns may be altered.
\end{abstract}

\section{INTRODUCTION}

Current day emergency services rely for data communications on public radio networks like GPRS. Sometimes in disaster situations, even GSM is used for voice communication between relief workers. However, in case of emergency the public networks may get overloaded. So, the use of generally available public networks is not considered to be reliable enough for emergency situations. Moreover, GSM/GPRS is an infrastructure based network, highly susceptible to disasters in small and medium sized urban areas.

Consider the C2000 system [1] which is rolled-out today, it is based on TETRA (see table I). One may observe that there are limited data communication facilities available. Also this network is infrastructure based (there are fixed base stations) and thus susceptible to the type of disasters we have in mind (see below).

So, the state-of-the-art system lacks capabilities (e.g. in offered data rates or video support) and is not disaster proof. We need some form of infrastructure-less wireless networking and we need radio spectrum for it. What we could do is claim radio resources for our purpose and design the disaster-relief network using these resources ... However, the radio spectrum is fully allocated, although not always utilized (as observed

\begin{tabular}{|l|l|}
\hline Parameter & Value \\
\hline Frequency Range & $380-400 \mathrm{MHz}$ \\
Channel Spacing & $25 \mathrm{kHz}$ \\
TX Power (mobile) & $1-3 \mathrm{~W}$ \\
Channel Access method & TDMA \\
Modulation method & $\pi / 4-\mathrm{DQPSK}$ \\
Channel bit rate & $36 \mathrm{kbit} / \mathrm{s}$ \\
Maximum data rate (gross bit rate) & $28.8 \mathrm{kbit} / \mathrm{s}$ \\
Net data rate, non-protected: & $\mathrm{n} \times 7.2 \mathrm{kbit} / \mathrm{s}$ \\
Low-protected & $\mathrm{n} \times 4.8 \mathrm{kbit} / \mathrm{s}$ \\
High-protected & $\mathrm{n} \times 2.4 \mathrm{kbit} / \mathrm{s}$ \\
& (n $=1,2,3 \mathrm{or} 4)$ \\
Speech coding & A-CELP, $4.567 \mathrm{kbit} / \mathrm{s}$ \\
Range Rural & $\pm 14 \mathrm{~km}$ \\
Range Suburban & $\pm 4.5 \mathrm{~km}$ \\
\hline \multicolumn{2}{|c}{ TABLE I } \\
\hline \multicolumn{2}{|c|}{ RADIO PARAMETERS OF THE TETRA SYSTEM. }
\end{tabular}

in [2], [3], [4]). Moreover, disasters like severe industrial explosions do not occur often enough to allocate huge amounts of scarce radio resources exclusively for the relief services.

The ad-hoc networking scenario used in our Adaptive Adhoc Free band Wireless communications (AAF) project [5] is modelled after the disaster-relief scenario described in [6]. The scenario is based on the fact that small and medium-sized urban areas may be destroyed by natural disasters like tornadoes, by fires or by industrial accidents. A real-life example is in the S.E. Fireworks disaster in Enschede, the Netherlands (May 2000, [7]), where a fireworks depot exploded and destroyed a large part of the city, killing 23 people and injuring more than a thousand. In the first hectic hours after the last of the large explosions, the municipal disaster command in particular had great difficulty to gain an overview of the extent of the disaster, and of the situation in the disaster area. Fire brigade, police and relief workers in the medical chain experienced great communication problems, both internally and with one another, because transmission equipment appeared not to be working, or only inadequately. "When those involved had thought of using the national emergency network, it offered no solution" [7].

In such a scenario it is vital that communication between relief groups is established as quickly and as easily as possible. The fact that the infrastructure-based networks in such an area may be destroyed raises the need to communicate using infrastructure-less wireless technologies. So, a Radio Access 
Network (RAN) or some type of cellular network (GSM, UMTS) will not do. Access to the core telecommunications network will only be available at the periphery of the disaster area where the damage to the infrastructure is less severe. The area of operation of the emergency network is confined to the disaster area, which is, say, no larger than a circle with a 2.5 kilometer radius (comparable to the Enschede case). An adhoc networking approach will allow the relief groups to enter the disaster area and communicate with each other quickly. With slightly more effort the wireless ad-hoc network can be hooked up to the original core telecommunications network almost instantly. The relief network must be able to handle a number of different types of traffic to service its users. Required network transport capabilities can range from lowbandwidth voice and email communication to high-bandwidth video and data traffic. Video and voice are streaming types of traffic, providing a more or less predictable network load with latency and jitter as important QoS parameters. On the other hand, data traffic is of rather bursty nature and requires reliable transmission.

In short, the network must be able to handle a wide variety of signals and has to deal with large, possibly unpredictable amounts of data. And, large amounts of data require, loosely spoken, large amount of radio resources. Which are scarce, especially when prior fixed allocated. What to do?

The goal of the AAF project [5] is to research and demonstrate a Cognitive Radio system, which continuously adapts its communications scheme to the available resources. Cognitive Radio is defined as a radio that can change its transmission based on interaction with its environment [8]. This definition can be very broad, including knowledge of services, user behaviour and spectral usage. The AAF project focuses on the key element, i.e. searching for under-utilised spectrum, and (rapidly) adapting transmission accordingly.

The first step in the project is to focus on identification of free resources in the frequency domain and adaptive OFDM based transmission. We believe that the capacity to nullify individual carriers poses interesting opportunities for cognition, as was also observed in [3]. Important challenges include stability of the system, identification of interference to other systems and meta-communication (node discovery). All these aspects are addressed as part of the research.

Two validation platforms, capable of transmitting and receiving real-world radio signals, will prove the consistency and comprehensiveness of the solution. First, a "cognitive system" platform is foreseen that explores cognition aspects for disaster relief purposes at the application level using currentday Wireless Local Area Networking (WLAN) technology like IEEE 802.11 [9] and IEEE 802.16 [10] solutions. The second platform, a "cognitive radio system" aims at a physical layer (radio system) controlled by a MAC protocol layer, in which an adaptive baseband processing system and a spectral scanning system work together efficiently. The output of the second activity is targeted at standardization activities and radio-regulatory organizations, its aim is to show the technical feasibility of non-existent solutions, while the first activity explores the cognition aspects that are allowed by the currentday spectral allocation policies. In this paper ideas as to the second activity are presented.

This paper provides a motivated back-of-the-envelope node architecture with emphasis on the radio system. Aspects of the architecture will be researched during the course of the project (2004-2008).

In section II a short description of the disaster-relief network-system requirements is presented and its consequences for the radio system architecture are addressed. The radio system architecture is discussed in section III. Due to limitations posed by Analog to Digital Converters (ADCs) in the system, the wideness of the frequency range $(400 \mathrm{MHz}-$ $1000 \mathrm{MHz}$ ) that may be used fruitfully for relief networking and jamming-resilience requirements, some form of sweeping scanning-system and frequency-hopping OFDM system is foreseen.

The Bluetooth system [11] provides a solution to the adhoc networking problem, using the $2.4 \mathrm{GHz}$ ISM band. In section IV of this paper we describe our intention to use the MAC layer of this system (FH/TDD) outside its intended frequency band, how to add cognitive aspects to it and how to allow it to work together with an OFDM based baseband system.

System alternatives, research issues and (future) experiments following from this design will be presented.

\section{From Requirements to Radio System ARCHITECTURE}

In the AAF project a disaster-relief network requirementsanalysis was undertaken, partly based on literature [12], [13], [14], [15]. Here, only a limited set of requirements is presented pertaining to the Incident Area Network (IAN), the network, temporary in nature, which is created on an ad-hoc basis for a specific incident:

- The network needs to be of an ad-hoc type.

- Voice is pre-dominant.

- Robust communication.

- Short connection set-up times

- The IAN consists of vehicle-mounted antennas (vehicle nodes) and small mobile units carried by users (personal nodes).

- There shall not be a single point of failure.

- The system is resistant to jamming (intentional disturbance of the communication in the relief network, e.g. by looting gangs).

- In rural areas personal nodes communicate in a single hop over distances of $500 \mathrm{~m}$ with a data rate of $2 \mathrm{Mb} / \mathrm{s}$.

- Personal nodes need to be able to operate without recharging batteries for 48 hours.

- Vehicle speeds up to $150 \mathrm{~km} / \mathrm{h}$ need to be supported.

In [16] these requirements were translated to requirements to the radio system of a personal node. 


\begin{tabular}{|l|c|}
\hline Parameter & Value \\
\hline Topology & multi-hop \\
Modulation scheme & OFDM \\
$f_{\min }$ & $400 \mathrm{MHz}$ \\
$f_{\max }$ & $1000 \mathrm{MHz}$ \\
Transmit power & $30 \mathrm{dBm}$ \\
Minimum Channel SNR & $15 \mathrm{~dB}$ \\
Minimum $B$ & $1 \mathrm{MHz}$ \\
Max. doppler $f_{d}$ & $185 \mathrm{~Hz}(100 \mathrm{~km} / \mathrm{h})$ \\
\hline
\end{tabular}

TABLE II

RADIO SYSTEM PARAMETERS. [16].

The distance requirement, leading to the conclusion that low frequencies need to be used is counterbalanced by the antenna size necessary for these frequencies. This leads to a frequency range of $400-1000 \mathrm{MHz}$. With a bandwidth of $1 \mathrm{MHz}$ and a SNR of $15 \mathrm{~dB}$ the (AWGN) capacity of a single channel is approximately $5 \mathrm{Mb} / \mathrm{s}$. The modulation scheme was chosen to be OFDM, see table II. These requirements can be met with the radio system architecture below.

\section{A. Radio system architecture}

In figure 1 the functions of the radio system of a personal node in the IAN are shown. The radio system consists of a ba-
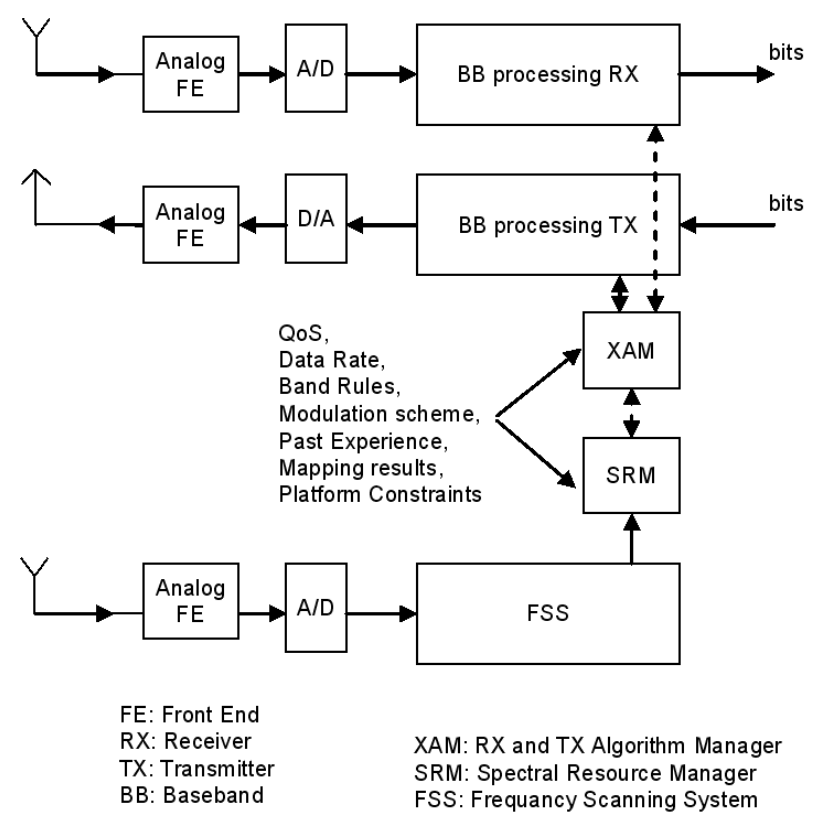

Fig. 1. Radio System Functions.

seband processing part (receiving BB-RX and transmitting BBTX) and a Frequency Scanning System (FSS). These functions operate on digital signals. Analog frontends (FEs) are used for channel selection and downconversion/upconversion of analog signals. Observe that it need not necessarily be so that the front-end of the baseband processing system and the FSS have the same capabilities. For spectrum measurements we measure (logarithmic) signal power, while for (de-)modulation purposes the exact signal shape is important. For the A/D and
D/A conversion quadrature conversion systems are foreseen. In the figure an $\mathrm{rX}$-and-tX Algorithm-Manager (XAM) function is depicted. Based on the items in the centre of the figure this algorithm manager selects an algorithm for a specific function or adapts parameters of an algorithm. The aim of the XAM is to provide a power-efficient radio system and to utilize reconfiguration capabilities of the computation platform (a System on a Chip (SoC)) [17]. Finally a Spectral Resource Manager (SRM) is depicted. While the FSS is a (simple?) frequency scanner, the SRM function is hosting "intelligence": it will, for instance based on past-experience [18], decide what usage will be made of the scanning information and guide the XAM to control the baseband processing.

Figure 1 shows all relevant functions, however in a reference-model like fashion, so not a particular instance of the architecture is shown, but all instances fit into this model. E.g. future research may lead to combining the receiving baseband processing signal chain with the FSS chain (thereby saving one analog frontend and an ADC). Also, in the figure it is suggested that both the RX and TX signal chains have separate antennas - which need not be the case in for instance a TDD based system.

\section{RADIO SYSTEM FUNCTIONS}

In this section we focus on three issues pertaining to the design of the radio system shown in figure 1:

- the role of the ADC in the system design,

- the parameters of the OFDM system in the baseband processing part, and

- three alternative scanning system architectures.

We will show that, due to the power consumption requirements of the personal node, severe limitations exist as to the channel bandwidth of the system that can be used for baseband processing and scanning. Given both the jamming-resilience requirements and the node-discovery problem in the project, a spread spectrum technique is considered necessary. We chose to add a form of frequency hopping to both the baseband processing system and the scanning system. Of course, this frequency hopping system is expected to be beneficial for the licensed users as well, as interference by the cognitive network might be made minimal.

\section{A. The Role of the AD Converter}

In our SDR project [19], in which we combined the physical layer of a HiperLAN/2 receiver with a Bluetooth one, we observed that the AD Converter was the most important single receiver part as far as power consumption was concerned. In fact, ADC design was used as a starting point for the entire project - a balance between power consumption of the analog frontend, the ADC and the (digital) baseband processing needed to be found. Also here we take the ADC as a pivotal point for our further research.

In order to compare the performance of analog-to-digital converters a Figure of merit (FoM) can be used that takes into account the converter's power dissipation $\mathrm{P}[\mathrm{W}]$, its 


\begin{tabular}{|c|c|c|c|c|}
\hline $\begin{array}{c}\text { Frequency } \\
\text { Range } \\
{[\mathrm{MHz}]}\end{array}$ & $\begin{array}{c}\text { Minimum } \\
{[\mathrm{dBm}} \\
/ 10 \mathrm{kHz}]\end{array}$ & $\begin{array}{c}\text { Maximum } \\
{[\mathrm{dBm}} \\
/ 10 \mathrm{kHz}]\end{array}$ & $\begin{array}{c}\Delta \mathrm{dB} \\
{[\mathrm{dBm}} \\
/ 10 \mathrm{kHz}]\end{array}$ & $\begin{array}{c}\mathrm{b} \\
{[\mathrm{bit}]}\end{array}$ \\
\hline $400-405$ & -120 & -75 & 45 & 9 \\
$406-420$ & -125 & -40 & 85 & 15 \\
$420-450$ & -110 & -70 & 40 & 8 \\
$450-470$ & -125 & -40 & 85 & 15 \\
$470-512$ & -125 & -35 & 90 & 16 \\
\hline
\end{tabular}

TABLE III

DENVER 1995 DATA IN THE $400 \mathrm{MHz}-500 \mathrm{MHz}$ RANGE [23]. resolution (specified in Effective Number of Bits (ENOB)) and its sampling rate $f_{s}[\mathrm{~Hz}],[20],[21]$. This FoM is given by

$$
\text { FoM }=\frac{P}{{ }_{2} \text { ENOB } \cdot f_{s}}[\mathrm{pJ} / \text { conversion-step }] \text {. }
$$

The data presented by Walden [22] can be extrapolated to the 2005 situation as is done is figure 2, in which each dot represents an ADC design. One may observe that a FoM of approximately $2 \mathrm{pJ} /$ conversion-step seems to be a reasonable assumption for today's ADCs. We now are able to relate

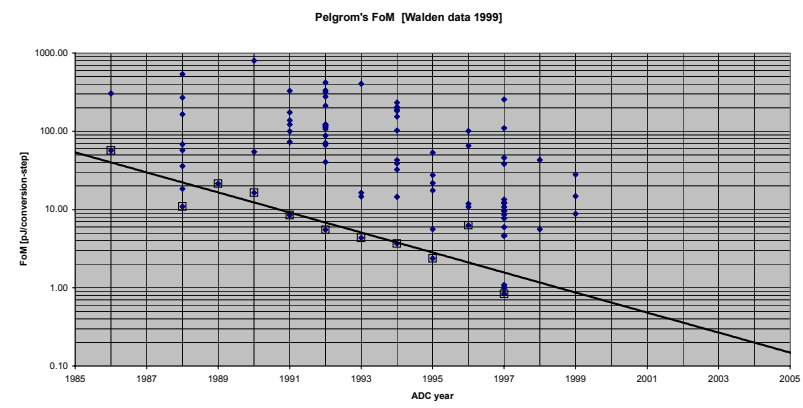

Fig. 2. Figure of merit (FoM) for ADCs.

the power consumption of an ADC to its required bandwidth and ENOB. As both the baseband processing system and the scanning system operate in an environment where licensed users operate, the analog frontends of these systems have to be able to cope with both strong and weak signals at the same time. The ENOB that an ADC must support can be estimated by the dynamic range $\Delta_{\mathrm{dB}}$ that occurs in the band of interest. To avoid non-linear behaviour, we want signals that are simultaneously present not to overflow the system. In [23] a measurement campaign in Denver, Colorado that took place in 1995 is presented. Results in the $400-500 \mathrm{MHz}$ region reduced to $10 \mathrm{kHz}$ bins are repeated here in table III. As can be seen, a dynamic range as high as $90 \mathrm{~dB}$ was reported.

In the recent New York spectrum survey [24, pp.77-84] a maximum dynamic range of $80 \mathrm{~dB}$ can be observed for the $400 \mathrm{MHz}-500 \mathrm{MHz}$ frequency range. Also, these numbers can be compared to the dynamic range capabilities of modernday spectrum analysers (like the Rohde\&Schwarz FSP3 or the Agilent E4407B) which is in the order of $80 \mathrm{~dB}$.

By applying a version of the $6 \mathrm{~dB} /$ bit-rule for $\mathrm{AD}$ converters (applicable for white noise),

$$
\Delta_{\mathrm{dB}} \approx-4.77+b \cdot 6.02,
$$

we relate the dynamic range $\Delta_{\mathrm{dB}}$ to the number of bits $b$ of an $\mathrm{AD}$ converter (and assume $\mathrm{ENOB}=b$ ), see the last column in table III. By combining (1), in which the bandwidth $B=f_{s} / 2$ was selected, with (2) figure 3 was made.

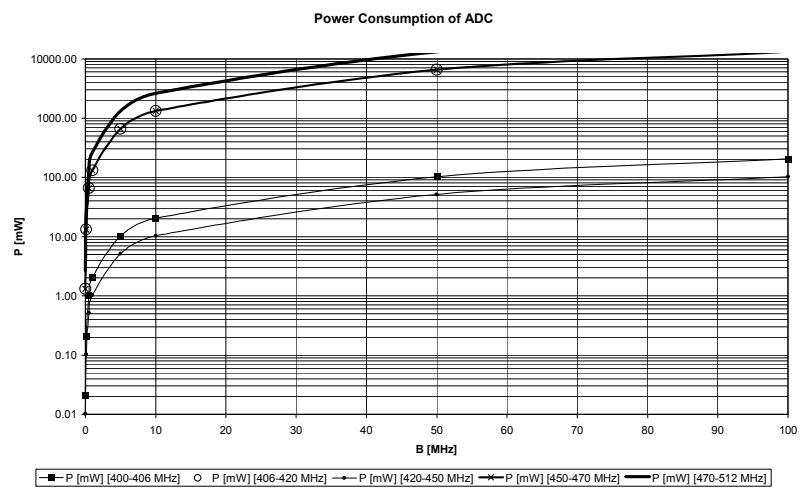

Fig. 3. Power consumption of an ADC as a function of bandwidth with dynamic range estimates taken from the Denver 1995 measurement campaign ( [23], assuming $2 \mathrm{pJ} /$ conversion-step and $f_{S}=2 B$ ).

Due to the large dynamic range present in a (part) of the band of interest and the power requirements of the AAF personal node only rather small channel bandwidths $(\ll 100 \mathrm{MHz})$ can be used in the baseband and in the scanning system. It may be obvious that the scanning system must be able to sustain a large dynamic range (as spectrum analysers do). However, the baseband processing system must sustain this range too as there is no specially made-available disaster-relief bandwidth reserved and high power peaks of legacy users may occur in the band used by the AAF system. So, only a channel bandwidth less than, say $\mathrm{B}=5 \mathrm{MHz}$, very likely only $\mathrm{B}=1 \mathrm{MHz}$, appears to be feasible.

Now, if one considers the jamming-resilience requirement mentioned in section II it seems obvious that a fixed one or five $\mathrm{MHz}$ wide band does not qualify. One could have thought that a true wideband ADC (say, supporting bandwidths larger than $100 \mathrm{MHz}$ ) would be an interesting feature providing jamming-resilience for a personal node in the IAN. However, the conclusion must be that this is not possible (observe that in the upper right corner of figure 3 the power of the ADC is larger than $10 \mathrm{~W}$ ). We therefor propose to use some form of frequency hopping as a means to adhere to the jammingresilience requirement, both for the baseband processing system and the scanning system.

\section{B. OFDM parameters for the AAF frequency range}

The parameters of an OFDM system can be found by applying the method described by van de Beek [25, p. 31]. As a starting point for the analysis three parameters need to be known: the channel bandwidth B, the delay-spread of the channel $\tau$ and the maximum doppler frequency $f_{d}$. In OFDM systems the size of the cyclic prefix (or guard time) $T_{g}$ has to be larger than the delay spread. Also, the length of the useful part $T_{u}$ of the OFDM symbol has to be far larger than the length of the cyclic prefix in order to limit the loss of data rate due to overhead. In IEEE 802.16 [10], the ratio between guard time and useful part can be selected from 


\begin{tabular}{|c|c|c|c|c|c|}
\hline \multicolumn{7}{|c|}{ Average delay spread (3 routes) $[\mu s]$} \\
\hline \hline $\begin{array}{c}\text { Exceed } \\
\%\end{array}$ & $\begin{array}{c}430 \\
{[\mathrm{MHz}]}\end{array}$ & $\begin{array}{c}1350 \\
{[\mathrm{MHz}]}\end{array}$ & $\begin{array}{c}2260 \\
{[\mathrm{MHz}]}\end{array}$ & $\begin{array}{c}5750 \\
{[\mathrm{MHz}]}\end{array}$ & $\begin{array}{c}\text { Source } \\
\text { in [26] }\end{array}$ \\
\hline $50 \%$ & 0.7 & 0.6 & 0.6 & 0.27 & table 8 \\
$10 \%$ & 1.2 & 1.1 & 0.68 & 0.68 & table 8 \\
$1 \%$ & 1.86 & - & - & 0.93 & fig. 18, \\
& & & & & B25, B26 \\
\hline
\end{tabular}

TABLE IV

DELAY SPREAD MEASUREMENTS [26].

\begin{tabular}{|c|c|c|c|}
\hline $\begin{array}{c}B=f_{s} \\
{[M H z]}\end{array}$ & $\mathrm{N}$ & $\begin{array}{c}\Delta f \\
{[k H z]}\end{array}$ & $\begin{array}{c}T_{u} \\
{[\mu s]}\end{array}$ \\
\hline 1.28 & 128 & 10 & 100 \\
5.12 & 512 & 10 & 100 \\
\hline
\end{tabular}

TABLE V

OFDM PARAMETERS: SAMPLE FREQUENCY AND SYMBOL DURATION.

$T_{g}: T_{u}=1: 4,1: 8,1: 16$ and 1:32. Van de Beek states that, for the useful part duration $T_{u}=N T$, with $\mathrm{N}$ the number of carriers in the OFDM symbol and $T=1 / f_{s}$ the sampling time, $N \gg \tau f_{s}=\tau B$ should hold, as the channel bandwidth $\mathrm{B}$ is assumed to be equal to the sample frequency $f_{s}$. On the other hand, van de Beek observes that the inter-carrier spacing $\Delta f=\frac{1}{N T}$ should be chosen much larger than the maximum doppler frequency: $f_{d} \ll \Delta f$ or $N \ll B / f_{d}$. So

$$
\tau B \ll N \ll \frac{B}{f_{d}}
$$

and van de Beek suggests as a possible design to take the geometric mean of the extreme values as a means to calculate $\mathrm{N}$ :

$$
N \cong \sqrt{\frac{\tau B^{2}}{f_{d}}}=B \cdot \sqrt{\frac{\tau}{f_{d}}}
$$

This number suffices for our purposes here (but is not necessarily optimal).

It now remains to establish values for the parameters above. Two possible channel bandwidths were given in section III-A: around one and five $\mathrm{MHz}$.

The maximum doppler frequency has to be determined for a carrier frequency in the high regions of the AAF frequency range, say $f_{c}=900 \mathrm{MHz}$, and the maximum velocity is taken to be $v=120 \mathrm{~km} / \mathrm{h}$ (see section II). Then $f_{d}=\frac{v}{c} f_{c}$ with $c$ the speed of light. We find $f_{d}=100 \mathrm{~Hz}$.

An estimate of the delay-spread in the $400 \mathrm{MHz}$ region can be obtained from delay spread measurements reported in [26], see table IV. As can be seen from the excess quantiles, the delay spread in the $400 \mathrm{MHz}$ region is approximately twice as large as the delay spread in the $5.7 \mathrm{GHz}$ region. Now, for the ease of computation, assume the delay spread to be $\tau=$ $1 \mu \mathrm{s}$ (below, we will see that the resulting cyclic prefix can be chosen far larger than the $1 \%$ excess quantile in table IV).

Application of (4) gives $N \cong B \cdot 10^{-4}$. By selecting a sample frequency in such a fashion that $N$ is a power of two and near one or five $\mathrm{MHz}$, we arrive at table V. Finally, alternatives for the guard time $T_{g}$ (or the number of samples in the prefix, $\left.N_{g}=T_{g} / T\right)$ need to be defined. For this the ratio's in [10] are used, see table VI.

\begin{tabular}{|c|c||c|c||c|c|c|}
\hline \multicolumn{2}{|c||}{$\begin{array}{c}f_{s}=1.28 \\
{[M H z]}\end{array}$} & \multicolumn{2}{c||}{$f_{s}=5.12$} \\
{$[M H z]$} & \multicolumn{3}{l|}{} \\
\hline $\mathrm{N}$ & $N_{g}$ & $\mathrm{~N}$ & $N_{g}$ & $T_{u}: T_{g}$ & $T_{g}[\mu s]$ & $T_{s}[\mu s]$ \\
\hline 128 & 32 & 512 & 128 & $4: 1$ & 25 & 125 \\
128 & 16 & 512 & 64 & $8: 1$ & 12.5 & 112.5 \\
128 & 8 & 512 & 32 & $16: 1$ & 6.7 & 106.7 \\
128 & 4 & 512 & 16 & $32: 1$ & 3.4 & 103.4 \\
\hline
\end{tabular}

TABLE VI

OFDM PARAMETERS: GUARD TIME AND SYMBOL DURATION.

The values found above are of the same order of magnitude as those used in a T-DAB system variant (e.g. bandwidth $B=1.5 \mathrm{MHz}$, symbol duration $T_{s}=1.246 \mathrm{~ms}$, guard time $T_{g}=246 \mu \mathrm{s}$, number of carriers $N=2048$, carrier frequency in the range of $174-230 \mathrm{MHz}$ ) and in a DVB-T system variant (e.g. useful part duration $T_{u}=224 \mu \mathrm{s}$, guard time $T_{g}=7,14,28,56 \mu \mathrm{s}$, number of carriers $N=2048$, carrier frequency in the range of $470-862 \mathrm{MHz}$ with a $8 \mathrm{MHz}$ channel spacing), so we have no reason to doubt the feasibility of the OFDM baseband system presented here.

Observe that the OFDM system can be used to avoid legacy users through nullification of carriers as was shown in case it is incorporated into a TDD scheme [3]. In the design presented here a "bin-size" of $\Delta f=10 \mathrm{KHz}$ is available - which is approximately the smallest frequency allocation unit used by the Dutch Radio Regulatory Office in the $400-500 \mathrm{MHz}$ range. We need to investigate whether this provides sufficient frequency resolution to avoid legacy users, while maintaining a $2 \mathrm{Mb} / \mathrm{s}$ data rate (see section II).

\section{Scanning System types}

For the design of the Frequency Scanning System (FSS) we turn to the architecural principles of a spectrum analyser. Basically, for a spectrum analyser, there are two architectures available: a scan-based architecture and a real-time FFT based architecture [27], [28], [29].

In a scan-based architecture a mixer is used to translate the signals in a frequency band of interest downwards for filtering and analysis. By "sweeping" -changing of the mixing frequency in subsequent time intervals- a wide frequency span can be analysed. This analyser only provides a view on a part of the time-frequency space, as only a single band is measured in a particular time interval.

In a real-time FFT based analyser the signals in a frequency band of interest are down converted to baseband, sampled and analysed by taking the FFT of this signal. For a given frequency span, the real-time FFT analyser can be considered to measure frequency bands in parallel, while a scan-based analyser measures in serial fashion. So, in case the required frequency span is smaller than the bandwidth of the ADC converter an FFT based analyser is generally providing more speedily its results than a scan-based architecture can. The FFT analyser is sometimes even called a real-time analyser [29], as, as soon as all the samples for the FFT are available, immediately the FFT can be computed. This analyser therefore provides a complete view of the time-frequency space.

However, if the required frequency span is larger than the bandwidth of the ADC, then also an FFT-based analyser needs 
to change its mixing frequency in subsequent time intervals, so a form of sweeping is needed. The analyser that both needs to sweep and uses an FFT can be considered a hybrid of the two basic architectural principles and is coined a scanning-FFT based analyser.

From the discussion in section III-A it is clear that also the the ADC in the scanning system will have limited bandwidth compared to the frequency span of interest, so that some form of scanning-FFT based FSS is to be foreseen. While in traditional spectrum analysers the path taken in the timefrequency space is fixed, this need not be so in the scanning system of the personal node. One can conceive of a system that uses radio-regulatory knowledge for the determination of an initial hopping pattern (during the time when the system awaits a disaster). Once in operation the FSS continuously adapts its hopping pattern based on previous measurements (of course, the hopping pattern for the baseband processing has to be the same for all nodes that want to communicate, the FSS need not use identical hopping patterns for all nodes).

In order to decide upon the initial hopping pattern for the FSS, we will use data from the fixed radio-spectrum observation system of the Dutch radio-regulatory office (Agentschap Telecom, [30]).

\section{Combining OFDM AND BLUETOOTh Functions}

An overview of issues in ad-hoc networking is given by [31]. In there it is stated that (p.17) "Communication protocols for cognitive radio remain an open issue". In the paper adhoc techniques based on IEEE 802.11 and IEEE 802.16 are surveyed, the MAC layers are CSMA/CA based.

However, when these systems are used in the frequency region we intend to use in our system, they need to use bandwidths that can be accommodated by current-day ADCs. So, only bandwidths can be used that are similar to the ones we arrive at in this paper $(1-5 \mathrm{MHz})$. A prior fixed band of that width can easily and intentionally be disturbed completely. So, the systems above may not abide by the jamming-resilience requirements of the personal node. We do not consider them further here.

It now remains to be determined how to incorporate the hopping OFDM system into a cognitive ad-hoc network. A direction to the problem is provided in [3] in which a HiperLAN/2 alike Radio Access network (RAN) is made spectrum aware by introduction of a measurement phase in its TDD scheme. This system is infrastructure-based: there is an access point connected to a fixed infrastructure and mobile terminals communicating with it. This topology is not directly applicable to an ad-hoc networking situation. However, by assigning the role of an access point to a particular (mobile) node and allow that mobile node to also be able to play the role of a mobile terminal, an ad-hoc system can be conceived. The resulting system could use nodes that are capable of playing a master and a slave role, as is done in Bluetooth. Still, this solution is not jamming resilient either.

However, the Bluetooth system [11], [32] provides a solution to the jamming-resilient ad-hoc networking problem, using the
2.4 GHz ISM band. Using experience from [19], we do not see (technical, as opposed to regulatory) objections to investigate whether the MAC layer of this system (FH/TDD) can be used outside its intended frequency band. We intend to use OFDM as modulation scheme instead of GFSK.

Different scenario's as to add a measurement phase into the TDD frame can be conceived, thereby adding cognition to a jamming-resilient ad-hoc network. As an example, consider figure 4 in which two different scenario's are depicted. The circle denotes that a node is listening in order to discover the presence of legacy users.

In the upper scenario both master and slave first listen to the environment, are assumed to "hear" the same legacy users and start transmitting and receiving using the appropriate choice of OFDM carriers. In lower scenario transmission and listening takes place in parallel (one listens to legacy users on another channel than on which one transmits in order to avoid self blocking). It are these type of scenario's we intend to study

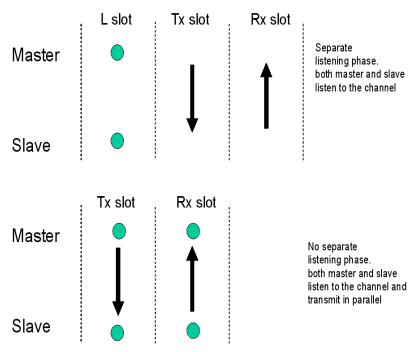

Fig. 4. Two scenario's for adding a measurement phase in the TDD scheme.

further in the course of our project, for instance in order to assess achievable data rates.

The first issue and main topic of this contribution, is however whether the OFDM symbols as defined in section III$\mathrm{B}$ fit in a Bluetooth-alike TDD frame and whether the required data-rates can be achieved (in case there are no legacy users, so all OFDM carriers may be loaded by the disaster-relief nodes).

\section{A. MAC Frame Duration, OFDM symbol length and burst duration}

In case no OFDM burst can be transmitted during the normal Bluetooth type of ad-hoc network set-up and maintenance, the possibility of more-or-less independent design of scanning system, network system and baseband system is at risk. So, we need to address this issue first.

As a starting point, we use the hopping rate that is used in the Bluetooth system, 1600 hops/s. In figure 5 the Bluetooth TDD frame is shown. It is not allowed to use the entire $625 \mu \mathrm{s}$ slot-duration for transmission purposes in the TDD frame, only approximately half of the slot duration may be used. In Bluetooth, after the bits in a slot are send, the system needs to hop to a new frequency. This takes some time as the synthesizer needs to stabilize itself on this new frequency. As can be seen approximately three $100 \mu$ s OFDM symbols (see table V) fit in this frame. So, with 800 master-slave 


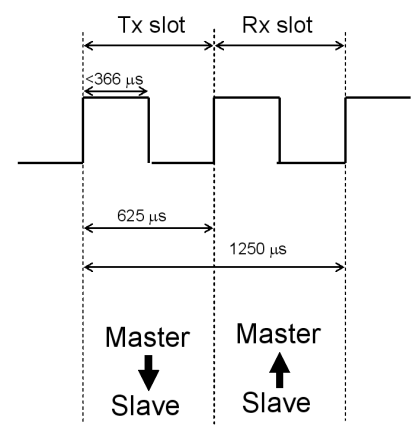

Fig. 5. RX/TX cycle of the Bluetooth system [11, p.85].

\begin{tabular}{|c|c|c|c|}
\hline Modulation & $b_{c}$ & $1.28 \mathrm{MHz}$ system & $5.12 \mathrm{MHz}$ system \\
\hline BPSK & 1 & 230400 & 921600 \\
QPSK & 2 & 460800 & 1843200 \\
16QAM & 4 & 921600 & 3686400 \\
64QAM & 6 & 1382400 & 5529600 \\
\hline
\end{tabular}

TABLE VII

MAXIMUM DATA RATE BETWEen MASTER AND SLAVE [BIT/S].

channel uses per second, we transmit 2400 OFDM symbols per second. For the $1.28 \mathrm{MHz}$ channel, 128 carriers are in the OFDM symbol, for the $5.12 \mathrm{MHz}$ channel there are 512 carriers (table VI). We assume that some of these carriers are used as guard carriers, and others as pilot symbols. We assume that the ratio between the total number of carriers $\mathrm{N}$ and the number of carriers used for data transmission purposes is identical to the ratio used in HiperLAN/2 [33]: a total of $N=64$ carriers of which 48 are data carriers. This means that if all carriers can be used (the band of interest is void of legacy users) 96 carriers can be loaded in the $1.28 \mathrm{MHz}$ system and 384 carriers can be loaded in the $5.12 \mathrm{MHz}$ system. In case of $b_{c}$ bits per carrier this amounts to $2400.96 . b_{c}$ bit/s in the $1.28 \mathrm{MHz}$ system and $2400.384 . b_{c} \mathrm{bit} / \mathrm{s}$ in the $5.12 \mathrm{MHz}$ system. The bit rates are shown in table VII. As can be seen, the data rates between master and slave do not match the $2 \mathrm{Mb} / \mathrm{s}$ requirements for the $1.28 \mathrm{MHz}$ system. On the other hand, for the 5.12 MHz system, QPSK suffices. As no further tweaking or optimization took place in the generation of these number, we believe that the system is worthwhile optimizing.

A few remarks need to be made. First, about the OFDM synchronization and channel estimation. In this example we assumed that three data symbols per slot can be sent, so we did not use a burst-preamble for timing synchronization purposes and frequency offset correction. Experiments have to be executed in order to establish whether this is feasible in real systems; possibly strong OFDM symbol synchronization needs to be used.

The data rates shown are raw rates, that is without error protective coding, framing etc.

Finally, the bit rates that can be reached in a radio environment in which legacy users are present need to be established.

\section{B. Issues and critical points}

In the original Bluetooth system, the hopping pattern (HP) is determined by the master node, basically this pattern is fixed (by the MAC address of that node). For the personal node in the IAN one could conceive a HP determined at IAN start-up (using the data of the radio regulation office). In this way location information can be used. Further, a HP may be investigated that adapts itself to the spectrum measurements, for instance in the case of too much legacy usage the network may decide to hop to another frequency.

A question is at what speed can be hopped and what frequency range can be covered in a single hop? What is the power consumption of the synthesizer that is necessary for this?

The issue of local versus distributed scanning needs to be addressed. Also, the issue of dissemination of spectral hole related information needs to be taken into account. Do all nodes in a piconet [32] see the same spectral usage? Can the master decide what OFDM carriers to use and assume that the slaves have "seen" the same spectrum holes? If so, they may expect what carriers will be used for the IAN (and no information needs to be transmitted). Here, the influence of temporal usage patterns of licensed users comes into play. A part of the AAF project consists of a spectral measurement campaign.

Finally, the frequency range of our hopping system must be supported by the analog frontend and antennas in the system. An antenna for a hopping range of $80 \mathrm{MHz}$ at $2.4 \mathrm{GHz}$ is possible (Bluetooth), what hopping range can be supported by a (simple) antenna system below $1 \mathrm{GHz}$ needs to be established.

\section{CONCLUSIONS AND FURTHER WORK}

In this paper we presented a node architecture for a personal node in a cognitive ad-hoc disaster relief network. This architecture is motivated from the network system requirements, especially single-hop distance and jamming-resilience requirements.

Due to limitations current-day ADCs, it seems necessary to use some form of Frequency Hopping Time Division Multiplexing (FH/TDD) system, similar to the Bluetooth system. It was shown that the OFDM symbol length for the frequency range at hand is consistent with a Bluetooth-alike FH/TDD scheme and provides (in some cases) the data-rates that follow from the requirements. Finally, we proposed to modify the FH/TDD scheme to incorporate a radio spectrum measurement phase.

In order to verify the system proposed here, a verification platform, the "cognitive radio system", will be built that implements the architecture in figure 1. The verification platform consists of two dual AMD Opteron PCs with PLDA PCI-X cards. One PC will be used as a transmitter, the other as a receiver. The PLDA cards are connected with an analog frontend via an a AD/DA board. The analog TX frontend provides both scanning and transmission, the analog RX frontend provides reception and scanning too. Scan frequency and carrier frequency for baseband processing can be selected independently. In this way we intend to demonstrate the functioning of the OFDM-based baseband processing in the $400-1000 \mathrm{MHz}$ range and the cooperation between baseband 
processing and scanning system. Different MAC frame scenarios (see section IV) and hopping scenario's will be tested with the platform. Measurements of achieved bit rates will be executed.

\section{ACKNOWLEDGMENT}

This work is co-sponsored by the Dutch Ministery of Economic affairs BSIK funding project and it is part of the FreeBand programme. The authors would like to thank their colleagues from THALES Communications in Huizen, the Twente Mobile and Wireless Centre (WMC), the International Research Centre for Telecommunications-transmission and radar (IRCT) of the Technical University Delft (TUD) and the Computer Architecture Design \& Test for Embedded Systems (CADTES) group at the University of Twente (UT) for the fruitful discussions in the AAF project.

For IEEE DySPAN

\section{REFERENCES}

[1] Dutch Ministry of Internal Affairs. "C2000 Website", 2004. http: //www.c2000.nl/.

[2] J. Mitola III. "Cognitive Radio for Flexible Mobile Multimedia Communications". Proc. International Workshop on Mobile Multimedia Communications, November 1999. also in appendix D of Mitola's PhD Thesis.

[3] T.A. Weiss and F.K. Jondral. "Spectrum Pooling: An Innovative Strategy for the Enhancement of Spectrum Efficiency". IEEE Radio Communications, 2004.

[4] M. McHenry. "Dynamic Spectrum Sharing". http://www. sharedspectrum.com/?section=presentations, January 2005.

[5] AAF. "The Adaptive Ad-hoc Free band communications (AAF) project Websites". http://www. freeband.nl/project.cfm? id=488, http://www.sas.el.utwente.nl/research/ wireless_communication/AAF/ and http://www.wmc. ewi.tudelft.nl/research/projects/aaf .htm, 2004-2008.

[6] J. Mitola III. Software Radio Architecture. John Wiley \& Sons, 2000.

[7] Commissie Oosting. "Final Consideration Commissie Vuurwerkramp Enschede", February 2001. http://www. minbzk.nl/contents / pages /4000/eindrapport_final_oosting_2-01.pdf.

[8] J. Mitola III. Cognitive Radio: An Integrated Agent Architecture for Software Defined Radio. $\mathrm{PhD}$ thesis, Royal Institute of Technology (KTH), Sweden, May 2000. http://www.it.kth.se/ j jitola/Mitola_Dissertation8_Integrated.pdf.

[9] IEEE. "802.11A-1999 / 8802-11, A (ISO/IEC 8802-11/Amd 1) Information Technology - Telecommunications and Information Exchange Between Systems - LAN/MAN - Specific Requirements”. IEEE Standard 802.11A-1999 (R2003), IEEE, 1999-2003.

[10] IEEE. "802.16-2004 IEEE Standard for Local and metropolitan area networks Part 16: Air Interface for Fixed Broadband Wireless Access Systems". IEEE Standard 802.16-2004, IEEE, 2004

[11] Bluetooth SIG. "Specification of the Bluetooth System - Core". Technical Specification Version 1.1, Bluetooth SIG, February 2001.

[12] R.E. Hunt (chairman). "Final Report of the Public Safety Wireless Advisory Committee". Report, PSWAC, September 1996.

[13] MESA Service Specification Group Services and Applications. "Statement of Requirements". Technical report, MESA, 2002.

[14] The SAFECOM Program. "Statement of requirements for Public safety Wireless Communications and Interoperability". Report, SAFECOM, March 2004. http://www. safecomprogram.gov/SAFECOM/ library/technology/1200_statementof .htm.

[15] EUROPCOM. "Mission/User requirements: specification and state of the art summary". Deliverable D2.1 - issue 1.0, November 2004. http://www.telecom.ewi.tudelft.nl/site/ research_details.html?id=59.

[16] N.A. Moseley. "Radio Resource Discovery for Ad-hoc Wireless Networking". Master's thesis, EECSM department, University of Twente, August 2004. EECSM-SAS17-04.
[17] J. Potman and F.W. Hoeksema. "The Adaptive Wireless Networking (AWGN) project web site". http://www.sas.el.utwente.nl/ index . php? fuseaction=research. showProject \&id=7, 2002-2005

[18] S. Haykin. "Cognitive Radio: Brain-empowered Wireless Communications". IEEE Journal on Selected Areas in Communications, 23(2):201220, February 2005.

[19] R. Schiphorst, F.W. Hoeksema, and V.J. Arkesteijn. "The BluetoothHiperLAN/2 SDR receiver project web site". http://www. sas.el. utwente.nl/home/SDR/, 2002-2005.

[20] M. Pelgrom. "Analog-to-digital Conversion", March 2003. Course Notes University of Twente, course 121048.

[21] K.H. Lundberg. "High-Speed Analog-to-Digital Converter Survey", October 2002. Accessible via http://web.mit.edu/klund/www/ papers/.

[22] R.H. Walden. "Performance trends for analog-to-digital converters". IEEE Communications Magazine, pages 96-101, February 1999.

[23] F.H. Sanders and V.S. Lawrence. "Broadband Spectrum Survey at Denver, Colorado". NTIA Report TR-95-321, NTIA, September 1995. http://www.its.bldrdoc.gov/pub/surv_dnv/.

[24] M. McHenry and D. McCloskey. "New York City Spectrum Occupancy measurements, September 2004". Report, Shared Spectrum Company, December 2004.

[25] J.J. van de Beek. Synchronization and Channel Estimation in OFDM Systems. PhD thesis, Luleå University of Technology, 1998. http://www.sm.luth.se/csee/sp/research/ thesis/bee98t.pdf.

[26] P. Papazian and M. Cotton. "Relative propagation Impairments between $430 \mathrm{MHz}$ and $5750 \mathrm{MHz}$ for mobile communication systems in urban environments". NTIA Report TR-04-407, NTIA, December 2003. http://www.its.bldrdoc.gov/pub/ntia-rpt/04-407/.

[27] Agilent. "Spectrum Analyser Basics". Application Note 150, Agilent, 2004.

[28] Agilent. "Swept and FFT Analysis". Performance spectrum analyzer series - application note, Agilent, 2004.

[29] Tektronics. "Fundamentals of Real-Time Spectrum Analysis". Technical report, Tektronics, 2004.

[30] Agentschap Telecom. "Web site of the Radiocommunications Agency in the Netherlands". http://www.agentschap-telecom.nl/, 2005

[31] I.F. Akyildiz, X. Wang, and W. Wang. "Wireless mesh networks: a survey". Computer Networks, 2004. Article in Press. Available at http: //www.ece.gatech.edu/research/labs/bwn/mesh.pdf.

[32] J.C. Haartsen. "Bluetooth - The universal radio interface for ad hoc, wireless connectivity". Ericsson Review, (3):110-117, 1998.

[33] ETSI. "Broadband Radio Access Networks (BRAN); HIPERLAN Type 2; Physical (PHY) layer". Technical Specification ETSI TS 101475 V1.2.2 (2001-02), ETSI, February 2001 